\title{
Three dimensional avalanche modelling across irregular terrain using DEM: Comparison with experiment
}

\author{
$\underline{\text { S. Mead }}^{a}$ and P. W. Cleary ${ }^{\mathrm{a}}$ \\ ${ }^{a}$ CSIRO Mathematics, Informatics and Statistics, Private Bag 33, Clayton South, VIC 3168, Australia \\ Email: Paul.Cleary@csiro.au
}

\begin{abstract}
Landslides are extremely destructive natural hazards that can cause significant economic damage and loss of life. Predicting the outcomes of landslides near settlements would prove useful for hazard mitigation and disaster management. Numerical simulation of landslides allows a wide range of outcomes based on different risks and mitigation strategies to be investigated at relatively low cost. For collisiondominated landslides, modelling using a continuum approach known as the Savage-Hutter method is most common. Another approach to modelling landslides is to use the discrete element method (DEM). This is a meshless numerical method which models the system at the particle level. It allows the prediction of individual collisions of particles with each other and with the surrounding terrain. DEM has previously been applied to landslide modelling using two dimensional discs (Cleary \& Campbell 1993, Campbell et al. 1995, Tang et al. 2009, Thompson et al. 2009) and three dimensional spheres (Cleary \& Prakash 2004). This approach neglects the important role particle shape plays in the behaviour of granular flow. The use of shaped particles in the form of super-quadrics for landslide modelling was first introduced by Cleary (2009, 2010). The non-sphericity of the particles was shown to have a strong impact on the flow, run-out distance and structure of the final deposit.
\end{abstract}

In this paper, DEM simulations are compared to laboratory-scale experiments of nominally dry sand avalanches across an irregular three dimensional terrain undertaken by Iverson et al (2004). Comparisons between the simulations and experiments are used to better quantify particle shape effects and to investigate the sensitivity of the method to material properties and numerical parameters.

Re-analysis of the published experimental results showed that the total volume of sand varied significantly during the avalanche, demonstrating strong dilation followed by weak compaction of the material. This has major implications for modelling since traditional Savage-Hutter models assume that the material is incompressible. Such dilation behaviour cannot be captured in this class of avalanche models. The advantage of the DEM approach is that it inherently captures such effects, which are heavily particle shape dependent. Critical information on material properties used in the experiment, such as particle shape, internal friction and cohesion, were not published in a quantitative manner suitable for use in DEM simulations.

The influence of material property uncertainties on DEM avalanche predictions was investigated by comparing the volume of sand retained in a reservoir at the top of the flume. The particle angularity and internal friction were found to have the most significant effect on the retained volumes, with the volume increasing as angularity and friction increased. The particle aspect ratios had less impact on the retained volume but were still important. A small envelope of material property ranges were identified which allowed the best prediction of the retained volume.

The final DEM deposit shapes within this optimal range of material properties were highly sensitive to the internal friction values. At an internal friction value of 0.46 , the DEM deposit had very similar features to the experimental deposits. At an internal friction value of 0.66 , resistance to the flow was too great and the deposits did not run far enough down the flume. At values of less than 0.36 the flow resistance was much lower, resulting in excessive run-out and lateral spread. These results indicate that a small increase in the internal friction of the DEM simulation from 0.46 is likely to closely match all the experimental attributes.

Keywords: Discrete Element Method (DEM), landslide, particle shape, experimental validation 


\section{INTRODUCTION}

Landslides are extremely destructive natural hazards that can cause significant economic damage and loss of life. A better understanding of landslide dynamics and run-out would enable better risk assessment, disaster management and hazard mitigation strategies; potentially decreasing the severity of the consequences of these events. Due to the unpredictable nature and variable scale of landslides, field measurements are usually limited to post-event surveys. These provide little guidance in hazard mitigation options such as the building of protective structures or relocation of at-risk infrastructure. In contrast, numerical simulation of landslides allows a wide range of risks and mitigation strategies to be investigated at relatively low cost. Accurate numerical models should, therefore, be considered a key component of managing landslide risk.

Numerical simulation of collision-dominated landslides such as rock avalanches and pyroclastic flows have been attempted using continuum mechanics models and granular flow models. Most continuum methods are based on two dimensional depth-averaged equations of motion similar to the shallow water equations and are collectively known as Savage-Hutter (SH) models (Savage and Hutter, 1989; Hutter et al 2005). The key assumptions of SH models are that the avalanche behaves as an incompressible, continuous media where the horizontal dimensions of the avalanche are much greater than the depth, and the height field remains single valued at all points.

Another approach to predicting outcomes of granular landslides is to use the Discrete Element Method (DEM). DEM is a meshless numerical method which models the system at the particle level. It predicts individual collisions between particles in the landslide and with the surrounding terrain. DEM was first applied to landslide modelling in two dimensions using a periodic section by Cleary \& Campbell (1993). They explored the effect of landslide mass on run-out over a simple two dimensional topography in Campbell et al. (1995). More recent examples of landslide modelling in two dimensions were given by Tang et al. (2009) and Thompson et al. (2009). DEM modelling of a landslide on real topography was extended to three dimensions by Cleary (2004) and Cleary \& Prakash (2004). In all of these examples, the DEM particles were modelled as round (discs in two dimensional and spheres in three dimensions). This neglects the important role that particle shape plays in the behavior of a granular flow as shown experimentally by Cho et al. (2006) who found that shear resistance and dilation increased as the particle angularity and aspect ratio were increased. The importance of particle shape was first highlighted using DEM by Cleary and Sawley (2002) in an investigation of discharge flows from hoppers. The use of non-round particles in the form of superquadrics (SQ's) for landslide modelling was introduced by Cleary $(2009,2010)$. They investigated the effect of shape using one of the landslide scenarios from Cleary and Prakash (2004). Particle shape was found to have a strong impact on the flow, the run-out distance and the structure of the final deposit. Cleary (2009) also provides examples of several industrial applications in which particle shape is important and an exposition of the role of particle shape and how it affects different types of particle flows. Owen et al. (2009) compared experimental results of slowly collapsing granular columns with two and three-dimensional DEM particles. This flow has much in common with landslides with the principle difference being velocity. The strength of the particle microstructure in two dimensional simulations (regardless of particle shape) was found to be too weak to correctly predict slope failure. Similarly spherical particles in three dimensions were also unable to correctly predict slope behavior. Including particle shape in three dimensions was found to increase the shear failure strength of the material allowing a reasonably accurate prediction of the shear planes which are critical in determining the lower boundaries of failing slopes. The same considerations apply to landslide prediction.

In this paper, DEM simulations of a laboratory scale landslide are compared to published experimental data. The process of using experimental data with uncertain material properties as the basis of numerical method validation is explored. Comparisons of the DEM and experimental results are also used to better quantify particle shape effects and investigate the sensitivity of the method to particle shape, material properties and numerical parameters on landslide predictions.

The DEM implementation used is a linear spring-dashpot model, described in detail by Cleary (1998, 2004). Super-quadrics, which have the general form:

$$
\left(\frac{x}{a}\right)^{m}+\left(\frac{y}{b}\right)^{m}+\left(\frac{z}{c}\right)^{m}=1
$$

are used to describe the shape of particles. The particle angularity is determined by the power $m$ with $m=2$ giving spherical particles and with the shape becoming cubic as $m \rightarrow \infty$. The aspect ratios are determined by the ratios of the semi-major axes ratios $b / a$ and $c / a$. 


\section{LABORATORY SCALE EXPERIMENT}

\subsection{Experimental configuration}

Iverson et al. (2004) performed bench top flume experiments of dry sand avalanches across an irregular three dimensional terrain in order to test a SH numerical model. The model of the flume is shown in Figure 1. It was $0.2 \mathrm{~m}$ wide, $\sim 1.0 \mathrm{~m}$ long and was fitted with a formed insert which created the irregular terrain. This was designed to promote complicated avalanche behaviour such as convergence, divergence and stranding of static material. A reservoir was fitted at the head of the flume to hold the granular material. This had an angled base with $33^{\circ}$ inclination which spanned the entire width of the flume. A $12 \mathrm{~cm}$ wide central opening on the reservoir was initially kept closed by a gate. There was also a small $(\sim 0.2 \mathrm{~mm})$ step at the interface between the reservoir and the terrain insert which

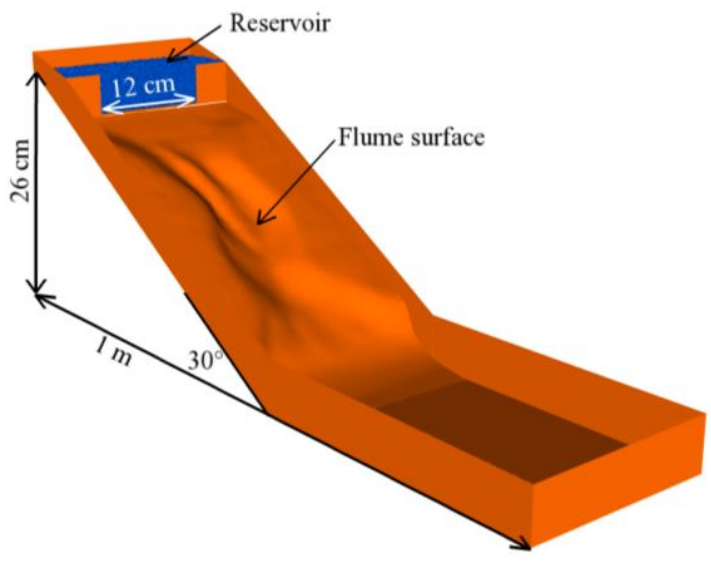

Figure 1. Flume configuration used in the DEM simulations, based on digitized contours of Iverson et al. (2004). Particles are initially located behind the reservoir at the top of the flume. caused some stranding of the granular material.

Angular and relatively homogenous grains of quartz sand with nominal diameters of 0.5-1.0 mm were used in the experiment. Published photomicrographs of the sand were used to quantify the size and shape of the particles. The major axis length of the sand was in the range 1.0-2.0 mm with aspect ratios of $0.54-1.0$. The friction angle of the sand on the terrain material was determined using a tilting table test to be $19.9^{\circ}$ giving particle-wall friction of 0.36 . A volume of $308 \mathrm{~cm}^{3}$ of the sand was poured into the reservoir and graded to form a flat surface. Avalanche flow was initiated by quickly raising the gate using a spring-loaded release mechanism. The restricted reservoir opening caused some material to remain stranded in the reservoir. This was designed to trap sand behind the retaining walls and force the sand to develop its own stable shape once the gate was removed, similar in nature to the self-formed boundaries of natural avalanches.

\subsection{Interpretation of experimental results}

Measurements of the three dimensional sand surface at several times during the experimental avalanche were provided by Iverson et al. (2004). Preliminary investigations of these measurements indicated that the rate and total volume of material discharged from the reservoir are the most important factors in determining the final shape of the stranded particle mass retained in the reservoir and of the avalanche deposit at the bottom of the flume. The discharge is controlled by the nature of the failure in the reservoir which is controlled by both the reservoir geometry and the strength of the granular material. In order to correctly predict the final deposit it is necessary to predict the correct volume of particles being released from the reservoir.

In the experiment, $26 \%$ of the granular material was left stranded in the reservoir. The slope of the reservoir floor affects the total volume of material retained, with steeper slopes causing more sand to drain from the reservoir. The aperture width limits both the discharge rate and the fraction of the volume discharged, as larger apertures increase the area available for the material to flow through while also reducing the volume of material trapped behind the retaining walls. Flow down the slope is also restricted by the step at the interface between the terrain and the reservoir. This step caused a small amount of material to be stranded behind it in the reservoir; around $0.5 \%$ of the total sand volume.

The strength of the granular material is controlled by the particle shape, friction and to a lesser degree by particle size. More extreme particle shapes (high angularity and aspect ratios) will increase the shear resistance of sand. Particles with higher angularities and more extreme aspect ratios will therefore have much steeper angles of repose, which will decrease the total volume discharged from the reservoir. Friction between the particles determines the ability of the grains to slide against each other. Higher values of interparticle friction increase the shear resistance and angle of repose, reducing the total volume discharged.

Figure 2 shows the time variation of total volume of sand, the volume of sand retained in the reservoir and the volume on the flume surface. The retained volume (dashed line) decreases with time and can be used to infer the discharge from the reservoir opening. Once the gate is opened, sand rapidly flows from the reservoir. In the first $1 \mathrm{~s}, 45 \%$ of the initial volume is discharged from the reservoir. It then slows markedly after $1 \mathrm{~s}$. All movement from the reservoir opening has ceased by $8 \mathrm{~s}$. Overall, $227 \mathrm{~cm}^{3}$ of sand has been discharged with $26 \%$ of the initial sand volume left stranded behind the reservoir walls. The volume of 
material on the flume surface (dotted line) increases with time as sand is discharged from the reservoir opening. This volume increases by approximately $348 \mathrm{~cm}^{3}$ in the first $1 \mathrm{~s}$. The rate that sand flows out into the flume then decreases substantially. At $8 \mathrm{~s}$, the volume on the flume surface is 417 $\mathrm{cm}^{3}$, which is almost double the volume discharged from the reservoir. The total volume of sand in the experiment (solid line) is the sum of the retained volume and volume of material on the flume surface. The total volume of sand is not constant throughout the experiment, meaning that the material does not behave incompressibly. The total volume increases sharply from its initial packed value of $308 \mathrm{~cm}^{3}$ to a peak of $512 \mathrm{~cm}^{3}$ at 1

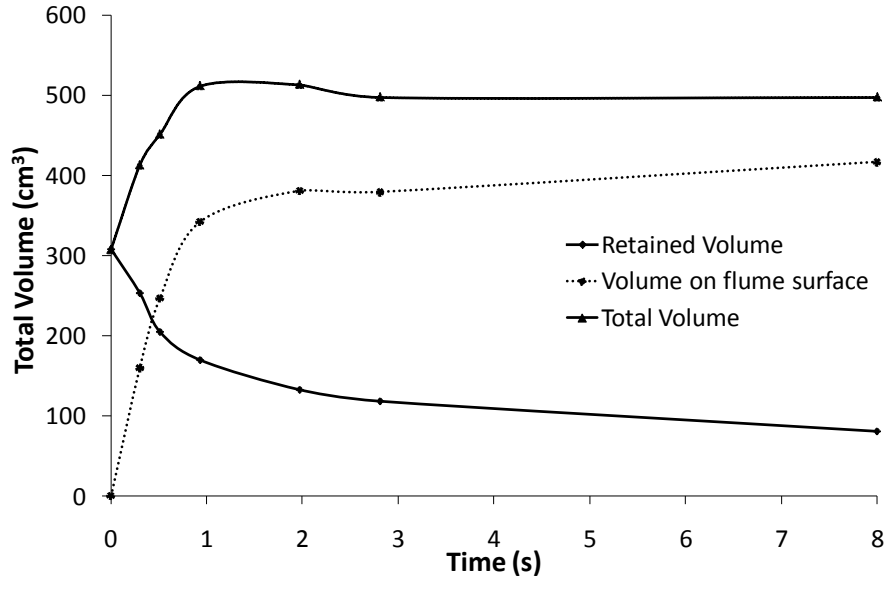

Figure 2. Variation with time of the total sand volume, volume behind the gate and volume on the flume surface. $\mathrm{s}$. It then decreases for the next $2 \mathrm{~s}$ to approximately $500 \mathrm{~cm}^{3}$ and remains constant until $8 \mathrm{~s}$.

The increase in the total volume means that there is significant dilation of the material during the experiment. Dilation occurs over the first second as the avalanche is initiated and the leading edge accelerates down into the flume. After $1 \mathrm{~s}$, the material compacts slightly as it comes to rest at the bottom of the flume, reducing the total volume. As the particle mass dilates due to shear during the early part of the flow, its volume increases thereby reducing the solid fraction (the ratio of the solid volume of material to the volume of space it occupies). The solid fraction is observed to have decreased from 0.48 (well packed) at the start of the experiment to only 0.3 (loosely packed) at the end. This large change in the solid fraction of the sand during the experiment indicates that the avalanche is clearly not incompressible. This has major implications for modelling since most models, such as $\mathrm{SH}$, assume that the flow is incompressible and the significant dilation behaviour in these avalanches cannot be captured. This results in large errors in deposit volume and probably in their shape and location. This may be one of the reasons for the disparity between the experimental data and the SH model by Iverson et al. (2004).

\subsection{Characterising undefined experimental material attributes}

While the material data provided by Iverson et al. (2004) is a useful input into numerical models, there are significant gaps. The basal friction, the initial volume and mass of sand are identified and well defined in the paper. However, other critical information such as the particle shape, internal friction, any cohesion and details of the step are not provided in a quantitative manner suitable for use in DEM simulation.

The internal friction angle of sand was reported as $43.99^{\circ}$, but this is not particularly useful since it is a bulk property resulting from a mixture of particle shape, surface friction and cohesion effects. This is commonly but erroneously equated to the inter-particle coefficient of friction which is required by the DEM contact model. The high surface roughness of the sand grains suggests that it should be higher than the basal coefficient of friction (which was 0.36 ). The sensitivity of the DEM model predictions to the specific value is uncertain and it is unclear how much higher the particle-particle coefficient of friction should be. The particle size and humidity during the experiment also means that there could have been some humidity-induced micro-liquid bridge cohesion. This would also increase the overall strength of the granular material and reduce discharge volumes. The effect of cohesion will be considered in future work.

The largest uncertainty in the data surrounds the quantification of shape. Photomicrographs of the sands used in the experiment were provided. However only one aspect ratio from each particle can be obtained since the photo only shows two dimensions. It is therefore unclear what range of values would adequately describe the second aspect ratio. The angularity of the grains is also uncertain but to a lesser degree since the curvature of the grains is visible. The particle angularity appeared to be in the range of 2.0 to 6.0. However, with only 25 full particles in the photograph, it is unclear how representative this sample actually is. Errors in the estimation of the particle shape will lead to inaccurate predictions of packing fraction and microstructure strength which will be reflected in errors in the angle of repose, the retained mass of particles in the reservoir and in the final deposit shape, size and location.

The uncertain inputs of particle aspect ratio, angularity and internal friction can have a significant effect on DEM predictions of the avalanche. In order to quantify the effects of these parameters, DEM simulations at 
varying particle shapes, sizes and internal friction values were run to find the range of values that predict a similar shape and reservoir-retained volume as the experimental results. This range provides an envelope of possible material conditions that discharge the correct amount of material from the reservoir, and which can be investigated further by comparing final deposits between the simulations and experiment.

\section{DETERMINING THE SIGNIFICANCE OF UNDEFINED MATERIAL PROPERTIES}

Table 1 displays the effect that particle size, angularity, aspect ratio and internal friction have on the volume retained behind the gate. We chose the major axis of the DEM particles to be larger than the experimental particle size at 3-4 $\mathrm{mm}$ or 2-3 $\mathrm{mm}$ to reduce computation time. The effect of particle size on the final deposit shape will be investigated further in future work. Rounded (2.2-3.0), moderately angular (2.5-4.0) and highly angular (2.5-6) particle shapes were chosen to represent the wide range of angularities seen in the experimental photomicrographs. The highest plausible particle aspect ratios based on the photomicrographs was 0.9-1.0/0.8-0.95 and the lowest range was 0.7-1.0/0.55-0.8. An intermediate range of 0.8-1.0/0.65-0.8 was also tested since most of the particle aspect ratios lie somewhere within this range. The coefficient of restitution was set to 0.5 for all simulations as initial tests showed little variation from this value. The numerical parameter of spring stiffness also made little difference to the results provided it was higher than $200 \mathrm{Nm}^{-1}$. This value gave average overlaps of less than $1 \%$ throughout the simulation.

Table 1. Variation in particle size, shape and internal friction of the volume retained behind the gate.

\begin{tabular}{|c|c|c|c|c|c|c|}
\hline Size (mm) & Angularity & $\begin{array}{c}\text { Case } \\
\text { Number }\end{array}$ & $\begin{array}{l}\text { Aspect } \\
\text { Ratio 1 }\end{array}$ & $\begin{array}{l}\text { Aspect } \\
\text { Ratio } 2\end{array}$ & $\begin{array}{l}\text { Internal } \\
\text { Friction }\end{array}$ & $\begin{array}{c}\text { Retained Volume } \\
\left(\mathrm{cm}^{3}\right)\end{array}$ \\
\hline \multirow{17}{*}{$3-4$} & \multirow{3}{*}{$2.2-3.0$} & 1 & $0.7-1$ & $0.55-0.8$ & 0.36 & 28.3 \\
\hline & & 2 & $0.8-1$ & $0.65-0.8$ & 0.36 & 25.2 \\
\hline & & 3 & $0.9-1$ & $0.8-0.95$ & 0.36 & 23.5 \\
\hline & \multirow{7}{*}{$2.5-4.0$} & 4 & $0.7-1$ & $0.55-0.8$ & 0.26 & 34.3 \\
\hline & & 5 & $0.8-1$ & $0.65-0.8$ & 0.26 & 38.0 \\
\hline & & 6 & $0.7-1$ & $0.55-0.8$ & 0.36 & 39.6 \\
\hline & & 7 & $0.8-1$ & $0.65-0.8$ & 0.36 & 34.2 \\
\hline & & 8 & 0.9-1 & $0.8-0.95$ & 0.36 & 37.3 \\
\hline & & 9 & $0.7-1$ & $0.55-0.8$ & 0.46 & 45.4 \\
\hline & & 10 & $0.8-1$ & $0.65-0.8$ & 0.46 & 40.8 \\
\hline & \multirow{7}{*}{$2.5-6.0$} & 11 & $0.8-1$ & $0.65-0.8$ & 0.26 & 46.1 \\
\hline & & 12 & $0.7-1$ & $0.55-0.8$ & 0.36 & 56.5 \\
\hline & & 13 & $0.8-1$ & $0.65-0.8$ & 0.36 & 57.0 \\
\hline & & 14 & $0.9-1$ & $0.8-0.95$ & 0.36 & 45.4 \\
\hline & & 15 & $0.8-1$ & $0.65-0.8$ & 0.46 & 58.6 \\
\hline & & 16 & $0.7-1$ & $0.55-0.8$ & 0.66 & 156.6 \\
\hline & & 17 & $0.8-1$ & $0.65-0.8$ & 0.66 & 119.0 \\
\hline \multirow{2}{*}{$2-3$} & \multirow{2}{*}{$2.5-4$} & 18 & 0.7-1 & $0.55-0.8$ & 0.66 & 34.6 \\
\hline & & 19 & 0.8-1 & $0.65-0.8$ & 0.66 & 28.0 \\
\hline
\end{tabular}

The results from Table 1 show the retained volume consistently increases as the particle angularity increases. This is consistent with the observations of the experiments run by Iverson et al. (2004) where the less angular sand travelled further down the flume and had a much larger lateral spread. Particles with a high angularity have also previously been shown by Cleary (2000) to significantly affect flow competency in DEM simulations of a dragline bucket filling with non-round particles. The aspect ratio has much less effect on the retained volume compared to the particle angularity. As the aspect ratios decrease from $0.9-1 / 0.8-0.95$ to 0.8 $1.0 / 0.65-0.8$ the retained volume increases for the rounded and high angularity cases but decreases in the medium angularity cases. The decreasing aspect ratio increases the number of neighbours that each particle is in contact with, resulting in higher shear strength of the material. A further decrease of the aspect ratio to 0.7$1.0 / 0.55-0.8$ also increases the retained volume in most cases. Two exceptions to this observation are shown from case 5 to 4 and 13 to 12 (Table 1), where the retained volume decreases by a small amount. This kind of behaviour was also observed in dragline bucket filling (Cleary, 2000) and was due to the preferential alignment of the particles with their long axis parallel to the flow allowing freer flow of particle layers over each other. The volume retained in the reservoir increases with higher values of internal friction due to the increased resistance to flow. Notably, the retained volume shows a very high sensitivity to friction at values higher than 0.46 , with the retained volume almost doubling when the value was increased to 0.66.

The volume of the material retained in the reservoir in the experiment was about $80 \mathrm{~cm}^{3}$. The high angularity cases gave the closest match to the experiment in terms of the retained material shape and volume. Case 17, with an internal friction of 0.66 retains too much material in the reservoir while case 15 retains too little with a friction value of 0.46 . It is likely that a friction value between these two cases would lead to the retention of 
the correct amount of material in the reservoir, leading to a more accurate prediction of the mass of the down flume deposit. This provides a small envelope of material properties that were investigated further by comparing with the down flume deposit.

\section{COMPARISON OF EXPERIMENTAL AND DEM MODEL DEPOSITS}

The transient progression of the avalanche down the flume for case 15 is shown in Figure 3 . This case gave the best match to the experimental deposit shape within the envelope of conditions giving a good representation of the retained volume. The effect that the terrain has on the flow is shown through the convergence, separation and stranding of material on the flume surface. At $0.3 \mathrm{~s}$, the leading edge of the avalanche is travelling faster than $1 \mathrm{~m} / \mathrm{s}$ and has split into two streams due to a bump in the terrain that separates the flow. The leading edge of the avalanche reaches the bottom of the flume and slows to $0.2 \mathrm{~m} / \mathrm{s}$ by $0.7 \mathrm{~s}$. Some material is still flowing from either side of the reservoir opening. At $2.8 \mathrm{~s}$, most of the material is now stationary, but there are two small streams still flowing from the sides of the opening which come to rest on the deposit at the bottom of the flume. A small volume of material is stranded just below the reservoir opening where two ridges restrict the flow. All motion in the flume ceases by $8 \mathrm{~s}$. Most of the material ends up in the deposit at the bottom of the flume, with a smaller amount retained in the reservoir and stranded below the opening.

The final simulated deposit (Figure 3d) is very similar to the experimental one (at $8 \mathrm{~s}$ ). The DEM deposit starts about $4 \mathrm{~cm}$ further down the flume and has $3 \mathrm{~cm}$ more lateral spread than the experimental deposit. A cross sectional profile of the final deposit surfaces for the experiment and DEM case 15 is shown in Figure 4. They are closely matched almost everywhere. Between 0.45 and $0.5 \mathrm{~m}$ there is slightly less material in the simulated deposit than was recorded in the experiments, but the difference is minor at less than 1 particle diameter. The greatest difference occurs in a small region at the start of the deposit, between 0.3 and $0.33 \mathrm{~m}$, where the DEM deposit is $1-2$ particle diameters lower (thinner) than the experimental one. This indicates that the resistance to flow is slightly too low. Higher resistance to flow would also decrease the lateral spread of the deposit and cause it to come to rest further up the flume.

Comparisons between all cases simulated showed that the final deposit was highly sensitive to the internal friction coefficient. At an internal friction value of 0.36 , material flowed freely from the reservoir opening and the final deposit started at approximately $0.4 \mathrm{~m}$. However, for internal friction of 0.66 , most of the material was stranded on the flume surface just below the reservoir. As a result, the down flume deposit was much smaller and did not spread as much as the case shown in Figure 3. These final deposit results show a high sensitivity to the internal friction value and indicate that conditions that will give the best prediction of the experimental avalanche surface lies within a small range of inter-particle friction values for particles with the shape used in case 15 .

\section{CONCLUSION}

Published experimental data of a laboratory-scale avalanche by Iverson et al. (2004) was investigated and compared with DEM simulations of the avalanche. Uncertainties in the values of particle angularity, aspect ratio and internal friction due to gaps in the reported experimental data were quantified by simulating a large range of these parameters and comparing the shape and volume of material retained in the reservoir. The comparisons demonstrate the significance of particle shape in DEM simulations and showed that the particle
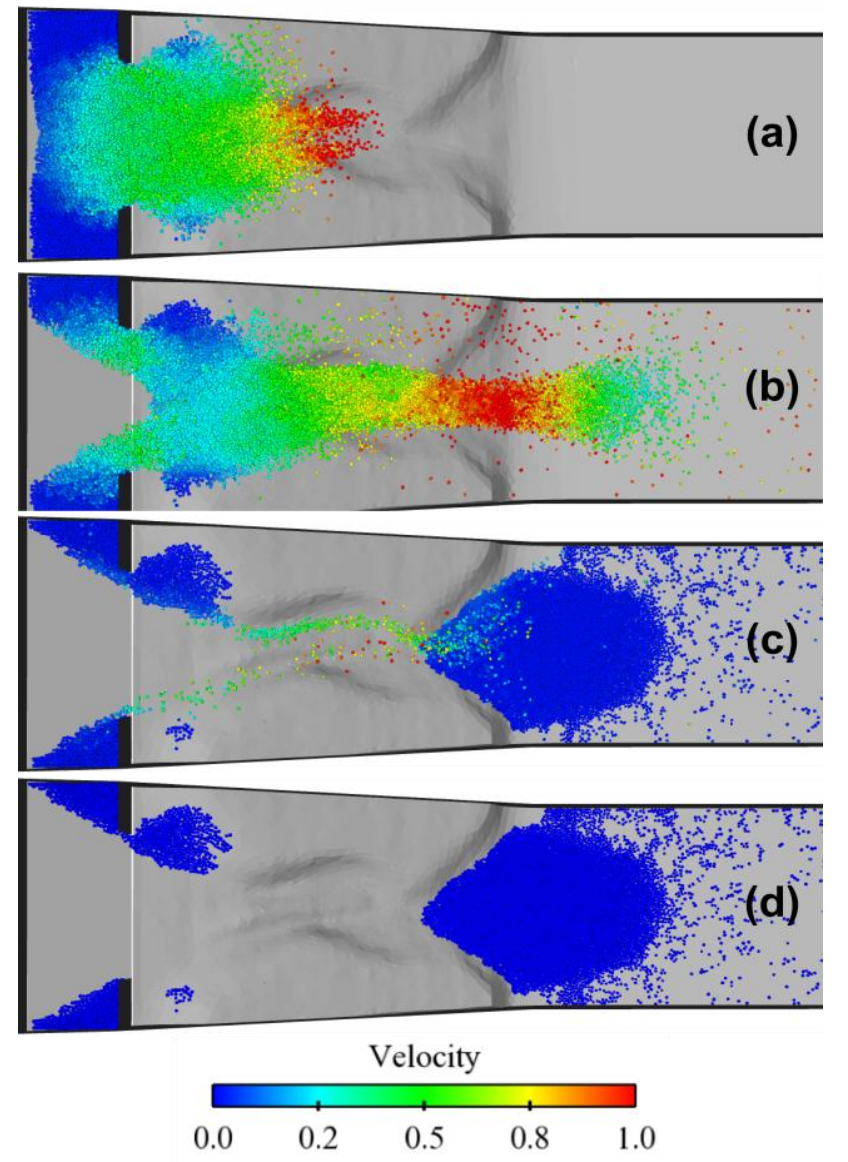

Figure 3. Progression of the avalanche down the flume for case 15 at: (a) $0.3 \mathrm{~s}$, (b) $0.7 \mathrm{~s}$, (c) $2.8 \mathrm{~s}$ and (d) $8.0 \mathrm{~s}$. 
angularity had a larger effect on the retained volumes in the reservoir than aspect ratio. The retained volumes were also highly sensitive to the value of internal friction, with higher values significantly affecting discharge from the reservoir. This highlights the importance of designing, thoroughly testing and reporting the operating conditions of validation experiments, since seemingly minor omissions in the reported experimental data can create large and unquantified uncertainties in the set-up of simulations, and consequently affect the model predictions. Comparisons of the final deposit shape between the simulated and experimental avalanche showed a high level of agreement, with the DEM deposit exhibiting slightly more lateral spread and starting slightly further down the flume. At higher values of internal friction, the DEM deposit shows less lateral spread and comes to rest further up the flume when compared with the experiment. This indicates that a small increase in the friction from case 15 is likely to closely match all the observations.

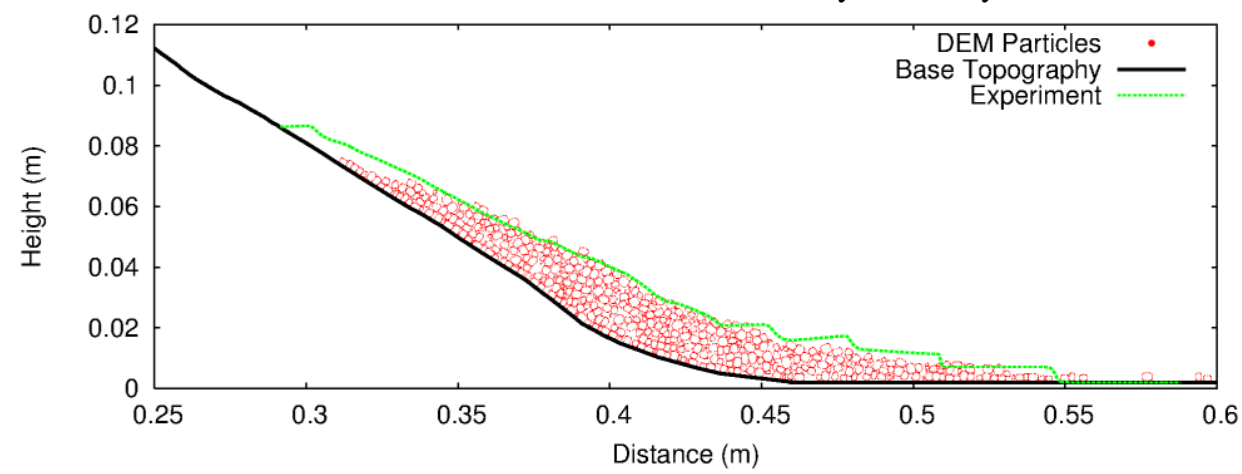

Figure 4. Final deposit depths along the centre line of the flume for the experiment and case 15.

\section{REFERENCES}

Campbell, C. S., Cleary, P. W. \& Hopkins, M. 1995. Large-scale landslide simulations: Global deformation, velocities and basal friction. J. Geophys. Res., 100, 8267-8283.

Cho, G-C., Dodds, J. \& Santamarina, J. C. 2006. Particle shape effects on packing density, stiffness and strength: Natural and crushed sands. J. Geotech. Geoenviron., 132(5), 591-602.

Cleary, P. W. \& Campbell, C. S. 1993. Self-Lubrication for Long Runout Landslides: Examination by Computer Simulation. J. Geophys. Res., 98, 21911-21924.

Cleary, P. W. 1998. Discrete element modelling of industrial granular flow applications. TASK Quarterly. 2.

Cleary, P. W. 2000. DEM simulation of industrial particle flows: case studies of dragline excavators, mixing in tumblers and centrifugal mills. Powder Technol., 109, 83-104.

Cleary, P. W. \& Sawley, M. L. 2002. DEM modelling of industrial granular flows: 3D case studies and the effect of particle shape on hopper discharge. Appl. Math. Model., 26, 89-111.

Cleary, P. W. \& Prakash, M. 2004. Discrete-element modelling and smoothed particle hydrodynamics: potential in the environmental sciences. Phil. Trans. R. Soc. A, 362, 2003-2030.

Cleary, P. W. 2004. Large scale industrial DEM modelling, Eng. Computation., 21, 169 - 204.

Cleary, P. W. 2009. Industrial particle flow modelling using discrete element method, Eng. Computation., 26(6), $698-743$.

Cleary, P. W. 2010. DEM prediction of industrial and geophysical particle flows. Particuology, 8, 106-118.

Hutter, K., Wang, Y. \& Pudasaini, S. P. 2005. The Savage-Hutter avalanche model: how far can it be pushed? Phil. Trans. R. Soc. A, 363, 1507-1528.

Iverson, R. M., Logan, M. \& Denlinger, R. P. 2004. Granular avalanches across irregular three-dimensional terrain: 2. Experimental tests. J. Geophys. Res., 109, F01015.

Owen, P. J., Cleary, P. W. \& Meriaux, C. 2009. Quasi-static fall of planar granular columns: comparison of 2D and 3D discrete element modelling with laboratory experiments. Geomech. and Geoeng., 4(1), 55-77.

Savage, S. B. \& Hutter, K. 1989. The motion of a finite mass of granular material down a rough incline. $J$. Fluid Mech., 199, 177-215.

Tang, C.-L., Hu, J.-C., Lin, M.-L., Angelier, J., Lu, C.-Y., Chan, Y.-C. \& Chu, H.-T. 2009. The Tsaoling landslide triggered by the Chi-Chi earthquake, Taiwan: Insights from a discrete element simulation. Eng. Geol., 106, 1-19.

Thompson, N., Bennett, M. \& Petford, N. 2009. Analyses on granular mass movement mechanics and deformation with distinct element numerical modeling: implications for large-scale rock and debris avalanches. Acta Geotech., 4, 233-247. 